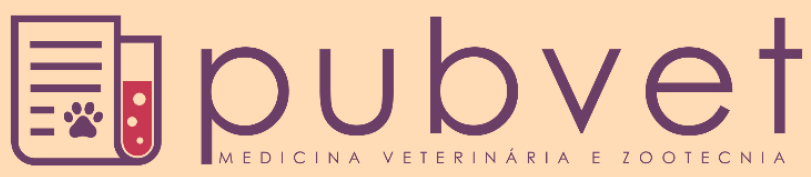

https://doi.org/10.31533/pubvet.v15n03a764.1-7

\title{
Cardiomiopatia dilatada arresponsiva ao tratamento com taurina em felino portador de insuficiência cardíaca congestiva direita
}

\author{
Leonardo Teixeira $^{1} \odot$, Aline Fukuda ${ }^{2}$, Talita Wajczyk ${ }^{1} \odot$, Juliana de Abreu Pereira ${ }^{3} \odot$ (D) Daina \\ Cristina Nehls Coutinho ${ }^{10}$
}

${ }^{1}$ Discente do curso de Medicina Veterinária, Universidade Sociedade Educacional de Santa Catarina, Joinville, Santa Catarina, Brasil. ${ }^{2}$ Médica veterinária na clínica Duhan Tamy's, Joinville, Joinville-Santa Catarina, Brasil.

${ }^{3}$ Docente do curso de Medicina Veterinária da Universidade Sociedade Educacional de Santa Catarina, Joinville-Santa Catarina, Brasil. *Autor correspondente: juliana.abreu@unisociesc.com.br

\begin{abstract}
Resumo. Na cardiomiopatia dilatada o miocárdio sofre degeneração progressiva, acarretando falha sistólica. Ocorre dilatação das câmaras cardíacas concomitante à diminuição da espessura das paredes dos ventrículos, o que ocasiona insuficiência das valvas atrioventriculares, redução da contratilidade do coração e o aumento do volume e do diâmetro sistólico final. Em estágios avançados se estabelece a insuficiência cardíaca congestiva, progredindo para congestão pulmonar e efusão pleural e pericárdica. Um felino, sem raça definida, castrado, pesando $4,9 \mathrm{~kg}$, sem histórico conhecido foi atendido em uma clínica veterinária. Encontrava-se alerta, dispneico, com aumento importante de volume abdominal, normotérmico, mucosas normocoradas e com sinais de dor na região abdominal. Ao ultrassom constatou-se líquido intra-abdominal anecogênico e arquitetura vascular hepática dilatada com trajetos preservados. No exame ecodopplercardiográfico havia insuficiência de grau importante na valva tricúspide, discreta efusão no pericárdio sem risco de tamponamento cardíaco e veia cava caudal com importante congestão com ascite cardiogênica e acentuada efusão no espaço pleural. A análise dos líquidos cavitários indicou efusão hemorrágica e transudato modificado. Os exames apontaram quadro de cardiomiopatia dilatada concomitante à insuficiência cardíaca congestiva direita. O relato descreve o tratamento estabelecido, o qual permitiu uma sobrevida de 48 dias após o diagnóstico da patologia. $\mathrm{O}$ animal foi arresponsivo à suplementação com taurina.
\end{abstract}

Palavras-chave: Cardiomiopatia dilatada, felino, taurina

\section{Dilated cardiomyopathy arresponsive to treatment with taurine in feline with right congestive heart failure}

\begin{abstract}
In dilated cardiomyopathy the myocardium undergoes progressive degeneration, resulting in systolic failure. Dilatation of the cardiac chambers occurs concomitantly with the decrease in the thickness of the walls of the ventricles, which causes insufficiency of the atrioventricular valves, reduction of the contractility of the heart and an increase in the volume and final systolic diameter. In advanced stages, congestive heart failure is established, progressing to pulmonary congestion and pleural and pericardial effusion. A feline, mixed breed, neutered, weighing $4.9 \mathrm{~kg}$, with no known history was seen at a veterinary clinic. He was alert, dyspnoic, with a significant increase in abdominal volume, normothermic, normal mucous membranes and with signs of pain in the abdominal region. Ultrasound showed an anechoic intra-abdominal fluid and dilated hepatic vascular architecture with preserved paths. On echocardiographic examination, there was insufficiency of major degree in the tricuspid valve, slight effusion in the pericardium without risk of cardiac tamponade and caudal vena cava with significant congestion with
\end{abstract}


cardiogenic ascites and marked effusion in the pleural space. Analysis of cavity fluids indicated hemorrhagic effusion and modified transudate. The examinations showed a picture of dilated cardiomyopathy concomitant with right congestive heart failure. The report describes the established treatment, which allowed a survival of 48 days after the diagnosis of the pathology. The animal was responsive to supplementation with taurine.

Key words: Dilated cardiomyopathy, feline, taurine

\section{Miocardiopatía dilatada sin respuesta al tratamiento con taurina en paciente felino con insuficiencia cardíaca congestiva derecha}

Resumen. En la miocardiopatía dilatada, el miocardio sufre una degeneración progresiva, lo que resulta en insuficiencia sistólica. La dilatación de las cámaras cardíacas se produce con una disminución del grosor de las paredes de los ventrículos, lo que provoca insuficiencia de las válvulas auriculoventriculares, reducción de la contractilidad del corazón y aumento del volumen y del diámetro sistólico final. En estadios avanzados, se establece insuficiencia cardíaca congestiva, progresando a congestión pulmonar y derrame pleural y pericárdico. Un felino, mestizo, castrado, de 4,9 $\mathrm{kg}$ de peso, sin antecedentes conocidos fue atendido en una clínica veterinaria. Se encontraba alerta, disnoico, con aumento significativo del volumen abdominal, normotermia, mucosas normales y con signos de dolor en la región abdominal. La ecografía mostró un líquido intraabdominal anecoico y una arquitectura vascular hepática dilatada con vías conservadas. Al examen ecocardiográfico se observó insuficiencia de mayor grado en la válvula tricúspide, leve derrame en el pericardio sin riesgo de taponamiento cardíaco y vena cava caudal con importante congestión con ascitis cardiogénica y marcado derrame en el espacio pleural. El análisis de los fluidos de la cavidad indicó derrame hemorrágico y trasudado modificado. Los exámenes mostraron un cuadro de miocardiopatía dilatada concomitante con insuficiencia cardíaca congestiva derecha. El informe describe el tratamiento establecido, que permitió una supervivencia de 48 días después del diagnóstico de la patología. El animal no respondió a la suplementación con taurina.

Palabras clave: Miocardiopatía dilatada, felina, taurina

\section{Introdução}

As cardiomiopatias são afecções do músculo cardíaco associadas à disfunção cardíaca e classificadas por aspectos morfopatológicos ou quanto à causa (Backschat et al., 2016; Silva et al., 2014). Na cardiomiopatia dilatada (DCM) o miocárdio sofre degeneração progressiva, acarretando em falha sistólica (Ferasin et al., 2003; Hemdon et al., 2002). Essa alteração permite a dilatação das câmaras cardíacas concomitante à diminuição da espessura das paredes dos ventrículos, o que ocasiona insuficiência das valvas atrioventriculares, redução da contratilidade do coração e o aumento do volume e do diâmetro sistólico final. Em estágios avançados se estabelece a insuficiência cardíaca congestiva (ICC), que progride para congestão pulmonar e efusão pleural e pericárdica (Mora et al., 2006).

Felinos com DCM apresentam-se letárgicos, hipotérmicos, desidratados e dispneicos. São observadas alterações nas auscultas cardíaca e pulmonar em caso de efusão pericárdica ou pleural (Mora et al., 2006). Os achados em exames radiográficos incluem cardiomegalia, efusão pleural, congestão pulmonar venosa e edema pulmonar. Se presente ICC direita avançada, hidroperitôneo e hepatomegalia são notados (Mora et al., 2006). Em estudo conduzido entre 1994 e 2001 com 106 felinos portadores de cardiomiopatia, Ferasin et al. (2003) constataram que 10,4\% dos pacientes foram diagnosticados com DCM, sendo a raça Domestic Short Hair a mais prevalente. Essa enfermidade foi diagnosticada em média aos 9 anos, sendo as fêmeas as mais afetadas.

Felinos com concentração sérica de taurina inferior à $100 \mathrm{nmol} / \mathrm{ml}$ podem ter como causa base da DCM a deficiência deste aminoácido. Trata-se essa afecção inicialmente com a suplementação de taurina (Backschat et al., 2016; Silva et al., 2014) independentemente da concentração sérica ou plasmática (Hambrook \& Bennett, 2012). 
Este relato descreve a evolução do quadro de DCM em um felino sem raça definida (SRD) concomitante a ICC direita arresponsivo à suplementação com taurina. São destacados os achados radiográficos, ultrassonográficos e ecodopplercardiográficos nos exames do paciente, assim como o tratamento ao qual o mesmo foi submetido.

\section{Relato de caso}

Um felino, SRD, castrado, pesando 4,9 kg foi levado à clínica veterinária no município de Joinville, SC para avaliação. O animal havia sido resgatado e seu histórico vacinal era desconhecido. No exame físico encontrava-se alerta, dispneico, com aumento importante de volume abdominal (Figura 1). Apresentava-se normotérmico, com mucosas normocoradas e sinais de dor abdominal à palpação. $\mathrm{O}$ animal foi internado para terapia hospitalar. Foram solicitados exames complementares como hemograma, bioquímica sérica, exames radiográficos, testes para imunodeficiência felina (FIV) e leucemia felina (FeLV), ultrassonografia e ecodopplercardiograma.

As cavidades torácica e abdominal foram avaliadas por exame radiográfico nas projeções laterolateral esquerda e direita, onde se evidenciou presença de líquido cavitário em toda porção abdominal (Figura 2, seta preta). No tórax havia aumento da radiopacidade nos lobos pulmonares sugestivos de efusão pleural. Ainda foram observadas opacidade dorsal ao esterno entre o quarto e sétimo espaço intercostal, o que limitou a visualização do coração e sua silhueta por sobreposição de fluido (efusão pericárdica/pleural) no aspecto ventral do tórax (Figura 2, seta branca). Aproximadamente $100 \mathrm{ml} \mathrm{de}$ líquido turvo amarelado foram removidos após abdominocentese e toracocentese.

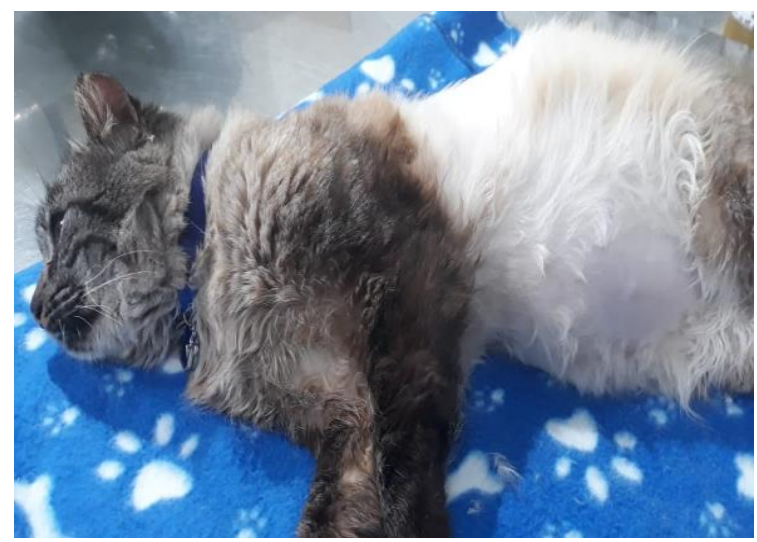

Figura 1. Registro no primeiro atendimento: paciente com aumento importante de volume abdominal.

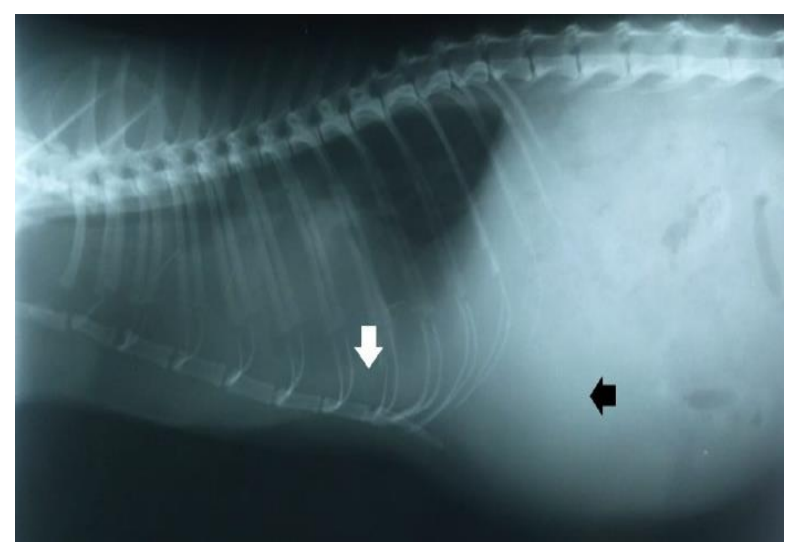

Figura 2. Imagem radiográfica na primeira consulta do paciente na projeção latero-lateral esquerda. Observase aumento da radiopacidade nas regiões abdominal (seta preta) e aspecto ventral do tórax (seta branca).

O hemograma (Tabela 1) evidenciou leucocitose, neutrofilia, linfopenia e trombocitopenia confirmada por esfregaço sanguíneo. A bioquímica sérica revelou hiperalbuminemia (Tabela 2), compatível com a desidratação do animal por efusões e processo congestivo. Os testes para FIV e FeLV foram negativos.

Durante o internamento, o animal apresentou efusão pleural, ascite e respiração abdominal. Foram administrados metronidazol ( $15 \mathrm{mg} / \mathrm{kg}$, a cada doze horas, IV) e ceftriaxona ( $25 \mathrm{mg} / \mathrm{kg}$, a cada doze horas, IV) devido à leucocitose; omeprazol $(0,5 \mathrm{mg} / \mathrm{kg}$, a cada doze horas, IV), tramadol (1 mg/kg, uma vez ao dia, IV), meloxican $(0,1 \mathrm{mg} / \mathrm{kg}$, uma vez ao dia, IV) e furosemida ( $4 \mathrm{mg} / \mathrm{kg}$, a cada oito horas, IV).

O exame ultrassonográfico evidenciou hepatomegalia moderada; fígado com contornos regulares, bordas finas e parênquima homogêneo com aumento discreto de ecogenicidade. Vasos hepáticos estavam dilatados e com trajetos preservados (Figura 3A). Havia presença de líquido livre abdominal anecogênico com partículas em suspensão, sugerindo efusão peritoneal moderada a importante (Figura 3B). Sem evidências de linfadenomegalia.

Amostras de líquido pleural e abdominal foram coletadas e enviadas para análise. O líquido pleural apresentou aspecto turvo, $\mathrm{pH} 7,5$ contendo proteínas $(2,6 \mathrm{~g} / \mathrm{dL})$, eritrócitos $\left(0,16 \times 10^{3} / \mathrm{mm}^{3)}\right.$ e leucócitos 
$\left(2.000 / \mathrm{mm}^{3}\right)$. A citologia revelou presença de neutrófilos segmentados $(21 \%)$, linfócitos (72\%), macrófagos (4\%), eosinófilos (3\%), presença de eritrofagocitose e ausência de microrganismos, sugerindo efusão hemorrágica. A presença de hemácias e leucócitos estaria relacionada com a dilatação dos vasos pelo processo congestivo, permitindo o extravasamento de conteúdo vascular.

Tabela 1. Resultado do exame de hemograma na primeira consulta. Equipamento modelo POCH-100-IV.

\begin{tabular}{lcc}
\hline Variáveis & Primeira consulta & Referências \\
\hline Eritrócitos & 7,56 & $5,0-10,0$ milhões $/ \mathrm{mm}^{3}$ \\
Hematócrito & 39,1 & $24-45 \%$ \\
Hemoglobina & 10,5 & $8-15 \mathrm{~g} / \mathrm{dL}$ \\
VCM & 45,1 & $39-55 \mathrm{fL}$ \\
CHCM & 30,8 & $30-36 \mathrm{~g} / \mathrm{dL}$ \\
Plaquetas & 76.000 & $200.000-500.000 / \mu \mathrm{L}$ \\
Leucócitos totais & 24.400 & $5.000-19.500 / \mu \mathrm{L}$ \\
Bastões & 0 & $0-300 / \mu \mathrm{L}$ \\
Segmentados & 21.228 & $2.500-12.500 / \mu \mathrm{L}$ \\
Neutrófilos & 21.228 & $2.500-12.800 / \mu \mathrm{L}$ \\
Monócitos & 976 & $0-850 / \mu \mathrm{L}$ \\
Linfócitos & 732 & $1.500-7.000 / \mu \mathrm{L}$ \\
Eosinófilos & 1.464 & $0-1.500 / \mu \mathrm{L}$ \\
Basófilos & 0 & Raros \\
\hline
\end{tabular}

Tabela 2. Resultado do exame de bioquímica sérica. Equipamento modelo Tekna VET 3000.

\begin{tabular}{lccc}
\hline Variáveis & Primeira consulta & Último retorno & Referência \\
\hline ALT & 23 & 45 & $10-80 \mathrm{U} / \mathrm{L}$ \\
AST & 83 & 24 & $10-88 \mathrm{U} / \mathrm{L}$ \\
Creatinina & 0,8 & 1,3 & $0,8-1,8 \mathrm{mg} / \mathrm{dL}$ \\
Albumina & 3,9 & - & $2,1-3,3 \mathrm{~g} / \mathrm{dL}$ \\
Ureia & - & 49 & $10-60 \mathrm{mg} / \mathrm{dL}$ \\
\hline
\end{tabular}

A análise de líquido abdominal indicou pH 7,0 contendo proteína $(4,13 \mathrm{~g} / \mathrm{dL})$, albumina $(2,4 \mathrm{~g} / \mathrm{dL})$, globulina $(1,23 \mathrm{~g} / \mathrm{dL})$ e relação albumina/globulina $(1,38)$. A contagem celular indicou eritrócitos $\left(0,02 \times 10^{3} / \mathrm{mm}^{3}\right)$ e leucócitos $\left(200 / \mathrm{mm}^{3}\right)$. A avaliação citológica evidenciou neutrófilos segmentados (99\%) e linfócitos (1\%) e ausência de microrganismos, sendo o líquido ascítico classificado como transudato modificado.
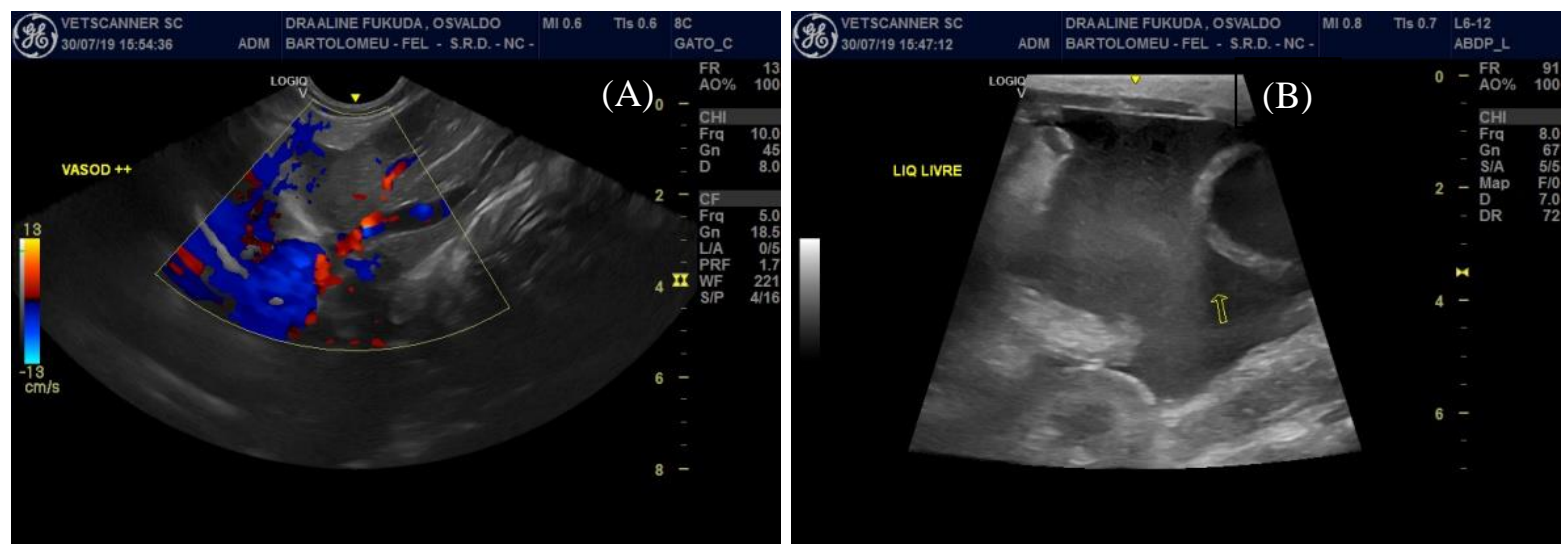

Figura 3. Imagens ultrassonográficas obtidas na primeira consulta: arquitetura vascular hepática dilatada com trajetos preservados (A) e com presença de líquido intra-abdominal anecogênico com partículas em suspensão (B).

No exame ecodopplercardiográfico constatou-se aumento moderado no diâmetro das cavidades esquerda (com diâmetro sistólico aumentado e diastólico normal) e direita. Foram observadas e insuficiência importante da valva tricúspide (Figura 4A) via Doppler, discreta efusão pericárdica (Figura 4B) sem risco de tamponamento cardíaco e aumento discreto da pressão sistólica e pulmonar. Ausência de trombos intracavitários. A veia cava caudal apresentava importante congestão, o que permitiu 
associar este achado com ascite cardiogênica e acentuada efusão no espaço pleural (Figura 4C). Os achados sugeriram quadro de cardiomiopatia dilatada.
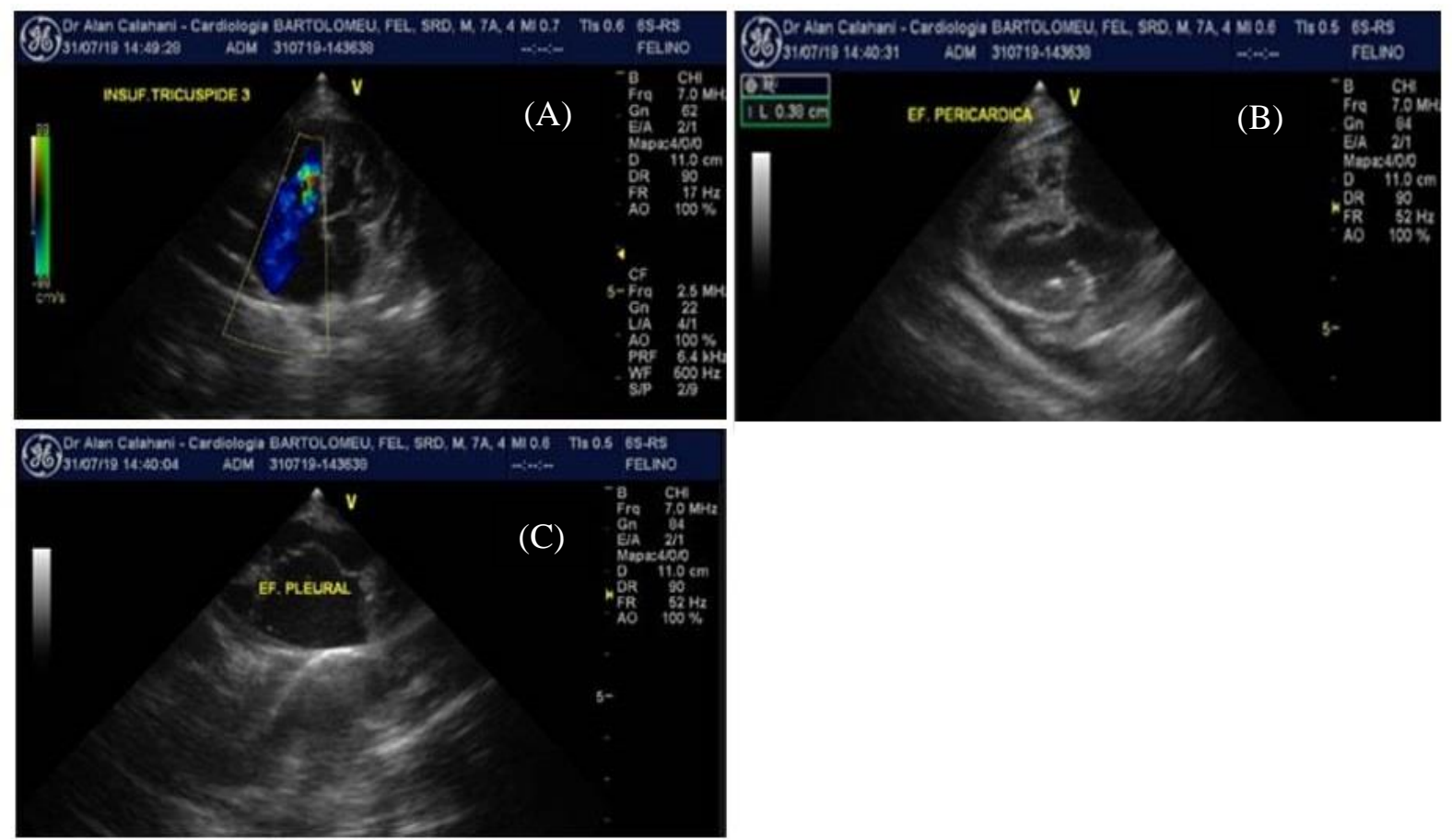

Figura 4: Imagens do exame ecodopplercardiográfico na primeira consulta: (A) insuficiência de grau importante na valva tricúspide, $(\mathbf{B})$ discreta efusão no pericárdio e $(\mathbf{C})$ veia cava caudal com importante congestão com ascite cardiogênica e acentuada efusão no espaço pleural.

Após a realização do exame, o paciente foi submetido à drenagem da efusão pleural. Foram prescritos para uso contínuo, por via oral, pimobendan $1,25 \mathrm{mg} /$ gato (a cada doze horas), clopidogrel $19 \mathrm{mg} / \mathrm{gato}$ (uma vez ao dia) e furosemida $1 \mathrm{mg} / \mathrm{kg}$; além da suplementação diária com taurina ( $250 \mathrm{mg} / \mathrm{gato})$. Com a estabilização do quadro, o animal foi liberado para tratamento domiciliar.

Trinta dias após alta médica, o animal retornou apresentando abaulamento abdominal. O paciente permaneceu sob observação durante o dia após abdominocentese, sendo liberado sem alta médica a pedido do proprietário. Foram mantidas todas as medicações previamente prescritas.

$\mathrm{O}$ animal retornou quinze dias após o segundo atendimento sob queixa de recidiva de ascite e efusão pleural (verificada por percussão). Em novos exames de hemograma e bioquímico (Tabela 2) verificouse trombocitopenia $(68.000 / \mu \mathrm{L})$, os outros parâmetros estavam dentro da normalidade.

O acompanhamento via exame ecodopplercardiográfico evidenciou aumento da dimensão da veia cava caudal, indicando que a causa da cardiomiopatia não era ocasionada por deficiência de taurina. Modificou-se a prescrição de furosemida para $4,5 \mathrm{mg} / \mathrm{gato}$ a cada doze horas e acrescentado ao protocolo sildenafil $1 \mathrm{mg} / \mathrm{kg}$ uma vez ao dia, administrado por via oral para melhorar a função ventricular direita. $\mathrm{O}$ animal foi liberado para casa após estabilização do quadro, vindo à óbito três dias depois. A necropsia não foi autorizada pelo proprietário.

\section{Discussão}

A caracterização de cardiomiopatia dilatada é estabelecida através do exame ecodopplercardiográfico. Segundo De Madron (2015), os achados incluem diminuição da inotropia com aumento das câmaras cardíacas, diminuição da espessura da parede ventricular, efusão pericárdica, redução da velocidade de ejeção aórtica e pulmonar, com possibilidade de refluxo nas válvulas mitral e/ou tricúspide. As características observadas ao exame ecodopplercardiográfico deste relato foram compatíveis com o descrito por De Madron (2015). A disfunção sistólica ventricular observada agravou o funcionamento do lado direito do coração, promovendo congestão da veia cava caudal, ICC direita, efusão pleural, ascite e, por fim, congestão generalizada. Embora o exame não tenha indicado a presença 
de trombos, suspeita-se que a trombocitopenia esteja relacionada à sequestro de plaquetas. Essa hipótese não pode ser comprovada pois a necropsia não foi autorizada.

Feldman et al. (2014) destacam estreita ligação entre hipertireoidismo e alterações cardíacas, passível de indução a distúrbios cardíacos como a cardiomiopatia dilatada. Eles relatam que 50\% dos animais acometidos por hipertireoidismo apresentam cardiomegalias, sendo os gatos idosos os mais acometidos. O paciente do presente relato não aparentava idade avançada ou sinais clínicos característicos do hipertireoidismo.

O paciente foi arresponsivo ao tratamento com taurina durante os 48 dias de acompanhamento. Conforme Hambrook \& Bennett (2012) há outras causas que conduzem ao quadro de DCM. A injúria no miocárdio pode ter causa viral, inflamatória, imunomediada, tóxica, metabólicas idiopáticas ou secundárias à infarto (Backschat et al., 2016; Silva et al., 2014). Todavia a causa não pode ser investigada via necropsia e exame histopatológico do miocárdio, como sugerido por Ferasin et al. $(\underline{2003})$.

Ferasin et al. (2003) reportam que a sobrevida média de felinos $(n=8)$ com DCM após o diagnóstico foi de 11 dias e apenas 25\% sobreviveram mais de 30 dias. Segundo Hambrook \& Bennett (2012) o tratamento para DCM arresponsiva à taurina é restrito ao uso de diuréticos, inibidores da ECA (vasodilatadores), toracocentese e oxigenoterapia. Os autores demonstraram que a sobrevida média pode chegar a 49 dias com a prescrição do pimobendan $(n=16)$, apresentando diferenciais como aumento da contratilidade do miocárdio e do débito cardíaco, mas sem aumentar a demanda de oxigênio. A digoxina, em contrapartida, não contribuiu significativamente para os pacientes testados por este autor.

A sobrevida do paciente após diagnóstico e início de tratamento foi compatível com o exposto na literatura (48 dias). O tratamento proposto fez uso ainda de clopidogrel para minimizar o risco de tromboembolismo arterial cardiogênico e, portanto, contribuir para a sobrevida do paciente (Hogan, 2017).

\section{Conclusão}

A DCM é uma patologia de baixíssima incidência em felinos, principalmente casos arresponsivos à suplementação com taurina e relacionados à machos. Estudos sobre as afecções de DCM em felinos, concomitantes ou não com ICC, são necessários para melhores condutas e aumento da sobrevida dos pacientes.

\section{Referências}

Backschat, P. S., Goldfeder, G. T., Ampuero, F., Lacerda, A. M. D., \& Larsson, M. H. M. (2016). Cardiomiopatia arritmogênica do ventrículo direito em felino: relato de caso. Arquivo Brasileiro de Medicina Veterinaria e Zootecnia, 68(5), 1112-1116. DOI: https://doi.org/10.1590/1678-4162-8616

De Madron, É. (2015). Evaluation of feline cardiomyopathies. In E. De Madron, V. Chetboul, \& C. Bussadori (Eds.), Clinical echocardiography of the dog and cat (pp. 207-228). Elsevier. DOI: https://doi.org/10.1016/b978-0-323-31650-7.00013-2

Feldman, E. C., Nelson, R. W., Reusch, C., \& Scott-Moncrieff, J. C. (2014). Canine and feline endocrinology. Elsevier Health Sciences.

Ferasin, L., Sturgess, C. P., Cannon, M. J., Caney, S. M. A., Gruffydd-Jones, T. J., \& Wotton, P. R. (2003). Feline idiopathic cardiomyopathy: a retrospective study of 106 cats (1994-2001). Journal of Feline Medicine and Surgery, 5(3), 151-159. DOI: https://doi.org/10.1016/s1098-612x(02)00133-x

Hambrook, L. E., \& Bennett, P. F. (2012). Effect of pimobendan on the clinical outcome and survival of cats with non-taurine responsive dilated cardiomyopathy. Journal of Feline Medicine and Surgery, 14(4), 233-239. DOI: https://doi.org/10.1177/1098612x11429645

Hemdon, W. E., Kittleson, M. D., Sanderson, K., Drobatz, K. J., Clifford, C. A., Gelzer, A., Summerfield, N. J., Linde, A., \& Sleeper, M. M. (2002). Cardiac troponin I in feline hypertrophic cardiomyopathy. Journal of Veterinary Internal Medicine, 16(5), 558-564.

Hogan, D. F. (2017). Feline cardiogenic arterial thromboembolism: prevention and therapy. Veterinary Clinics: Small Animal Practice, 47(5), 1065-1082. DOI: 0.1016/j.cvsm.2017.05.001 
Mora, J. M., Pérez, E., \& Millán, R. (2006). Trombo intraventricular izquierdo en un gato con cardiomiopatía dilatada. Clínica Veterinaria de Pequeños Animales, 26(2), 130-135.

Silva, L. A. P., Contieri, M. B., \& Ferreira, F. S. (2014). Cardiomiopatia arritmogênica do ventrículo direito do boxer-revisãode literatura. Revista Científica de Medicina Veterinária, 12(40), 128-138.

Histórico do artigo

Recebido: 2 de setembro de 2020 .

Aprovado: 13 de outubro de 2020.

Disponível online: 16 de janeiro de 2021.
Licenciamento: Este artigo é publicado na modalidade Acesso Aberto sob a licença Creative Commons Atribuição 4.0 (CC-BY 4.0), a qual permite uso irrestrito, distribuição, reprodução em qualquer meio, desde que o autor e a fonte sejam devidamente creditados. 